\title{
Microwave-induced magnetoresistance of two-dimensional electrons interacting with acoustic phonons
}

\author{
O. E. Raichev \\ Institute of Semiconductor Physics, National Academy of Sciences of Ukraine, Prospekt Nauki 41, 03028, Kiev, Ukraine
}

(Dated: November 24, 2018)

\begin{abstract}
The influence of electron-phonon interaction on magnetotransport in two-dimensional electron systems under microwave irradiation is studied theoretically. Apart from the phonon-induced resistance oscillations which exist in the absence of microwaves, the magnetoresistance of irradiated samples contains oscillating contributions due to electron scattering on both impurities and acoustic phonons. The contributions due to electron-phonon scattering are described as a result of the interference of phonon-induced and microwave-induced resistance oscillations. In addition, microwave heating of electrons leads to a special kind of phonon-induced oscillations. The relative strength of different contributions and their dependence on parameters are discussed. The interplay of numerous oscillating contributions suggests a peculiar magnetoresistance picture in high-mobility layers at the temperatures when electron-phonon scattering becomes important.
\end{abstract}

PACS numbers: 73.23.-b, 73.43.Qt, 73.50.Pz, 73.63.Hs

\section{INTRODUCTION}

Studies of high-mobility two-dimensional (2D) electron systems in weak perpendicular magnetic fields $B$ reveal several kinds of magnetoresistance oscillations which are caused by the scattering-assisted electron transitions between different Landau levels and survive increase in temperature $T$, in contrast to Shubnikov-de Haas oscillations. Most of attention is paid to the microwaveinduced resistance oscillations (MIRO) which appear under steady-state microwave (MW) irradiation of 2D electron gas. ${ }^{1}$ The MIRO periodicity is determined by the ratio of the radiation frequency $\omega$ to the cyclotron frequency $\omega_{c}$, while the amplitude is governed by the radiation power. As the power increases, MIRO minima are spread into intervals of zero dissipative resistance known as "zero-resistance states". ${ }^{2,3,4}$ The basic theoretical consideration ${ }^{5-9}$ of MIRO involves the picture of transitions between broadened Landau levels under elastic scattering of electrons by impurities or other inhomogeneities in the presence of MW excitation, when electrons absorb or emit radiation quanta. As a consequence of these transitions, both the electron scattering rate and the electron distribution function acquire oscillating components which lead to magnetoresistance oscillations. The corresponding contributions are often described in terms of "displacement" 5,6,7 and "inelastic" 8,9 microscopic mechanisms of MIRO, respectively. The above terminology emphasizes that the scattering-assisted MW absorption gives rise to spatial displacement of electrons along the applied dc field, while the modification of the distribution function is controlled by inelastic relaxation of electrons. Consideration of these mechanisms satisfactory explains both the periodicity and the phase of MIRO observed in experiments, as well as their power and temperature dependence. ${ }^{10,11,12}$

Inelastic scattering of electrons by acoustic phonons makes possible the phonon-assisted transitions of electrons between Landau levels. The probability of such transitions is maximal when the phonon momentum is close to the Fermi circle diameter, $2 p_{F}$. This leads to a special kind of magnetophonon oscillations also known as phonon-induced resistance oscillations (PIRO), ${ }^{13-17}$ whose periodicity is determined by commensurability of the characteristic phonon energy with cyclotron energy. In GaAs quantum wells with very high mobility $\left(\sim 10^{7} \mathrm{~cm}^{2} / \mathrm{V} \mathrm{s}\right)$, these oscillations are visible already at $T=2 \mathrm{~K},{ }^{17}$ while in the samples with moderate mobilities $\left(\sim 10^{6} \mathrm{~cm}^{2} / \mathrm{V} \mathrm{s}\right)$ they are well seen at $T=10-20 \mathrm{~K} \cdot{ }^{15}$ A theoretical description of PIRO assuming interaction of 2D electrons with anisotropic bulk phonon modes and showing a good agreement with experimental data has been presented recently. ${ }^{18}$

Both MIRO and PIRO are observed in the same interval of magnetic fields, $B<1 \mathrm{~T}$, and have comparable periods. Therefore, it is interesting to study the interplay of these remarkable oscillating phenomena. In other words, one may pose a question: what happens with magnetoresistance of the sample which shows PIRO if this sample is irradiated by microwaves? More generally, there exists an important problem of the influence of electron-phonon interaction on MW-induced magnetoresistance. Some aspects of this problem have been considered previously. First, it is well established that increasing MW power leads to heating of the electron system, especially in the samples with lower mobility. The effective electron temperature $T_{e}$ is determined by the power transmitted from electron system to the lattice via electron-phonon interaction. ${ }^{19}$ The heating causes a suppression of MIRO amplitudes ${ }^{11,20,21}$ for two main reasons: the enhancement of Landau level broadening and a decrease of the inelastic scattering time. In this sense, a consideration of electron-phonon interaction becomes necessary for description of MW photoresistance at elevated MW power. Further, as shown theoretically, ${ }^{22,23}$ there is a direct influence of electron-phonon scattering on the photoresistance: this scattering in the presence of MW excitation can give rise to pronounced magnetore- 
sistance oscillations whose behavior is different from that of the case of impurity-assisted transport. A consideration of electron-phonon interaction as a possible reason for the absolute negative resistivity and zero-resistance states has been also presented. ${ }^{23}$

The aim of this paper is to give a consistent theoretical description of magnetotransport under MW irradiation in the presence of both elastic and phonon-assisted electron scattering. It is demonstrated that the magnetoresistance has contributions of MIRO, PIRO, and the terms corresponding to interference of these types of oscillations. Apart from this, heating of electron system leads to an additional PIRO contribution which is shifted by phase with respect to equilibrium PIRO. Superposition of all these contributions gives rise to a complicated oscillating resistivity picture which is essentially different from either equilibrium magnetoresistance or MWmodified magnetoresistance under elastic scattering only. The analytical expression for magnetoresistance is obtained by assuming weak excitation, when the response is linear in MW power.

The paper is organized as follows. Section II describes the quantum Boltzmann equation for the system of $2 \mathrm{D}$ electrons interacting with impurities and phonons in the presence of microwaves. Section III is devoted to calculation of electron distribution function and resistivity. Section IV contains numerical results based on the expressions derived in Sec. III, description of different contributions to oscillating magnetoresistance, a discussion of the approximations used, and a brief conclusion.

\section{GENERAL FORMALISM}

In the region of weak magnetic fields, when the cyclotron energy is small in comparison with the Fermi energy, it is convenient to calculate the resistivity by using the quantum Boltzmann equation for the generalized Wigner distribution function $f_{\varepsilon \mathbf{p} t}$, which depends on en$\operatorname{ergy} \varepsilon$, quasiclassical 2D momentum $\mathbf{p}$ and time $t$. The coordinate dependence of this function is omitted, since only spatially-homogeneous 2D systems are under consideration. To take into account the influence of $\mathrm{MW}$ radiation and dc excitation on $2 \mathrm{D}$ electrons, one may use a transition to the moving coordinate frame (see Ref. 9 and references therein), when the MW and dc field potentials are transferred to the collision integrals and do not appear in the left-hand side of the kinetic equation. The scattering of electrons by impurities and phonons is treated within the self-consistent Born approximation (SCBA), which assumes that both the mean free path of electrons and the magnetic length are larger than the correlation lengths of the corresponding scattering potentials. Then, the quantum Boltzmann equation takes the form

$$
\left(\frac{\partial}{\partial t}+\omega_{c} \frac{\partial}{\partial \varphi}\right) f_{\varepsilon \mathbf{p} t}=J_{\varepsilon \mathbf{p} t}^{i m}+J_{\varepsilon \mathbf{p} t}^{p h}+J_{\varepsilon \mathbf{p} t}^{e e},
$$

where $J^{i m}, J^{p h}$, and $J^{e e}$ are the collision integrals for electron-impurity, electron-phonon, and electron-electron scattering, respectively. They are written as

$$
\begin{aligned}
J_{\varepsilon \mathbf{p} t}^{\kappa} & =-i \int d \tau e^{i \varepsilon \tau}\left\{\int d t ^ { \prime } \left[\Sigma_{t+\tau / 2, t^{\prime}}^{(\kappa) R}(\mathbf{p}) f_{t^{\prime}, t-\tau / 2}(\mathbf{p})\right.\right. \\
& \left.\left.-f_{t+\tau / 2, t^{\prime}}(\mathbf{p}) \Sigma_{t^{\prime}, t-\tau / 2}^{(\kappa) A}(\mathbf{p})\right]-\Sigma_{t+\tau / 2, t-\tau / 2}^{(\kappa)-+}(\mathbf{p})\right\}
\end{aligned}
$$

where $\kappa$ is the index of corresponding interaction $(\mathrm{im}$, $p h$, or ee). Throughout the paper, the system of units with $\hbar=1$ is used. The distribution function in the double-time representation, $f_{t_{1}, t_{2}}(\mathbf{p})$, is related to the Wigner distribution function $f_{\varepsilon \mathbf{p} t}$ by the Wigner transformation, which is defined for arbitrary function $A$ as $A_{\varepsilon \mathbf{p} t}=\int d \tau e^{i \varepsilon \tau} A_{t+\tau / 2, t-\tau / 2}(\mathbf{p})$. The Keldysh selfenergies are described by the following expressions:

$$
\begin{aligned}
\Sigma_{t_{1}, t_{2}}^{(i m)-+}(\mathbf{p}) & =-\int \frac{d \mathbf{q}}{(2 \pi)^{2}} w(q) e^{i \mathbf{q} \cdot \mathbf{R}_{t_{1} t_{2}}} G_{t_{1}, t_{2}}^{-+}(\mathbf{p}-\mathbf{q}), \\
\Sigma_{t_{1}, t_{2}}^{(p h)-+}(\mathbf{p}) & =-i \int \frac{d \mathbf{q}}{(2 \pi)^{2}} \int_{-\infty}^{\infty} \frac{d q_{z}}{2 \pi} \sum_{\lambda} M_{\lambda \mathbf{Q}} e^{i \mathbf{q} \cdot \mathbf{R}_{t_{1} t_{2}}} \\
& \times \int \frac{d \Omega}{2 \pi} D_{\lambda \mathbf{Q}}^{-+}(\Omega) e^{-i \Omega\left(t_{1}-t_{2}\right)} G_{t_{1}, t_{2}}^{-+}(\mathbf{p}-\mathbf{q}),(4)
\end{aligned}
$$

and

$$
\begin{array}{r}
\Sigma_{t_{1}, t_{2}}^{(e e)-+}(\mathbf{p})=-2 \int \frac{d \mathbf{q}}{(2 \pi)^{2}} U_{q}^{2} G_{t_{1}, t_{2}}^{-+}(\mathbf{p}+\mathbf{q}) \\
\times \int \frac{d \mathbf{p}^{\prime}}{(2 \pi)^{2}} G_{t_{1}, t_{2}}^{-+}\left(\mathbf{p}^{\prime}-\mathbf{q}\right) G_{t_{2}, t_{1}}^{+-}\left(\mathbf{p}^{\prime}\right) .
\end{array}
$$

The retarded and advanced self-energies, $\Sigma^{(\kappa) R}$ and $\Sigma^{(\kappa) A}$, can be expressed as

$$
\begin{gathered}
\Sigma^{(\kappa) R}=\Sigma^{(\kappa)-+}+\Sigma^{(\kappa)--}, \\
\Sigma^{(\kappa) A}=-\Sigma^{(\kappa)-+}-\Sigma^{(\kappa)++},
\end{gathered}
$$

where

$$
\begin{aligned}
& \Sigma_{t_{1}, t_{2}}^{(i m) \pm \pm}(\mathbf{p})=\int \frac{d \mathbf{q}}{(2 \pi)^{2}} w(q) e^{i \mathbf{q} \cdot \mathbf{R}_{t_{1} t_{2}}} G_{t_{1}, t_{2}}^{ \pm \pm}(\mathbf{p}-\mathbf{q}) \\
& \Sigma_{t_{1}, t_{2}}^{(p h) \pm \pm}(\mathbf{p})=i \int \frac{d \mathbf{q}}{(2 \pi)^{2}} \int_{-\infty}^{\infty} \frac{d q_{z}}{2 \pi} \sum_{\lambda} M_{\lambda \mathbf{Q}} e^{i \mathbf{q} \cdot \mathbf{R}_{t_{1} t_{2}}} \\
& \times \int \frac{d \Omega}{2 \pi} D_{\lambda \mathbf{Q}}^{ \pm \pm}(\Omega) e^{-i \Omega\left(t_{1}-t_{2}\right)} G_{t_{1}, t_{2}}^{ \pm \pm}(\mathbf{p}-\mathbf{q})
\end{aligned}
$$

and

$$
\begin{array}{r}
\Sigma_{t_{1}, t_{2}}^{(e e) \pm \pm}(\mathbf{p})=2 \int \frac{d \mathbf{q}}{(2 \pi)^{2}} U_{q}^{2} G_{t_{1}, t_{2}}^{ \pm \pm}(\mathbf{p}+\mathbf{q}) \\
\quad \times \int \frac{d \mathbf{p}^{\prime}}{(2 \pi)^{2}} G_{t_{1}, t_{2}}^{ \pm \pm}\left(\mathbf{p}^{\prime}-\mathbf{q}\right) G_{t_{2}, t_{1}}^{ \pm \pm}\left(\mathbf{p}^{\prime}\right)
\end{array}
$$


The quantities entering Eqs. (3)-(9) are described below. First, $G_{t_{1}, t_{2}}^{-+}(\mathbf{p})$ is the Keldysh Green's function for electrons, which is related to $f_{t_{1}, t_{2}}(\mathbf{p})$ as

$G_{t_{1}, t_{2}}^{-+}(\mathbf{p})=\int d t^{\prime}\left[f_{t_{1}, t^{\prime}}(\mathbf{p}) G_{t^{\prime}, t_{2}}^{A}(\mathbf{p})-G_{t_{1}, t^{\prime}}^{R}(\mathbf{p}) f_{t^{\prime}, t_{2}}(\mathbf{p})\right]$,

where $G^{A}$ and $G^{R}$ are the retarded and advanced Green's functions. The Green's function $G^{+-}$is expressed by the linear combination, $G^{+-}=G^{-+}+G^{R}-G^{A}$. Next, $D_{\lambda \mathbf{Q}}^{-+}(\Omega)$ is the Keldysh Green's function for phonons:

$$
\begin{aligned}
D_{\lambda \mathbf{Q}}^{-+}(\Omega) & =-2 \pi i\left[N_{\omega_{\lambda \mathbf{Q}}} \delta\left(\Omega-\omega_{\lambda \mathbf{Q}}\right)\right. \\
& \left.+\left(N_{\omega_{\lambda \mathbf{Q}}}+1\right) \delta\left(\Omega+\omega_{\lambda \mathbf{Q}}\right)\right],
\end{aligned}
$$

where $N_{\omega_{\lambda \mathbf{Q}}}=\left[e^{\omega_{\lambda \mathbf{Q}} / T}-1\right]^{-1}$ is the Planck's distribution function and $\omega_{\lambda \mathbf{Q}}$ is the phonon frequency. To find $G^{--}$, $G^{++}, D^{--}$, and $D^{++}$, one uses

$$
\begin{aligned}
G^{--}=G^{-+}+G^{R}, & G^{++}=G^{-+}-G^{A} \\
D^{--}=D^{-+}+D^{R}, & D^{++}=D^{-+}-D^{A} .
\end{aligned}
$$

The retarded and advanced Green's function of phonons are expressed as

$$
D_{\lambda \mathbf{Q}}^{R}(\Omega)=\frac{1}{\Omega-\omega_{\lambda \mathbf{Q}}+i 0}-\frac{1}{\Omega+\omega_{\lambda \mathbf{Q}}+i 0},
$$

and $D_{\lambda \mathbf{Q}}^{A}(\Omega)=\left[D_{\lambda \mathbf{Q}}^{R}(\Omega)\right]^{*}$. The retarded Green's function of electrons depends on the magnetic field $B$ and satisfies the equation

$$
\begin{gathered}
{\left[\varepsilon-\frac{\mathbf{p}^{2}}{2 m}+\frac{e^{2} B^{2}}{8 m c^{2}} \frac{\partial^{2}}{\partial \mathbf{p}^{2}}\right] G_{\varepsilon \mathbf{p} t}^{R}=1+\frac{1}{2} \int d \tau e^{i \varepsilon \tau} \int d t^{\prime}} \\
\times\left[\Sigma_{t+\tau / 2, t^{\prime}}^{(i m) R}(\mathbf{p}) G_{t^{\prime}, t-\tau / 2}^{R}(\mathbf{p})+G_{t+\tau / 2, t^{\prime}}^{R}(\mathbf{p}) \Sigma_{t^{\prime}, t-\tau / 2}^{(i m) R}(\mathbf{p})\right],
\end{gathered}
$$

where $m$ is the effective mass of electrons. The equation for the advanced Green's function $G^{A}$ differs from Eq. (14) only by a replacement of the superscripts $R$ with $A$. The integral on the right-hand side of Eq. (14) describes the influence of impurity scattering on the energy spectrum of 2D electrons in the magnetic field (the influence of other kinds of interaction on the electron spectrum is neglected here).

The electron-impurity interaction in Eqs. (3) and (7) is described by the Fourier transform of the random potential correlator, $w(q)$. The interaction with phonons [Eqs. (4) and (8)] is considered under approximations of equilibrium phonon distribution and bulk phonon modes. The influence of electron-phonon interaction on the phonon spectrum is neglected. The phonons are characterized by the mode index $\lambda$ and three-dimensional phonon momentum $\mathbf{Q}=\left(\mathbf{q}, q_{z}\right)$. The squared matrix element of electron-phonon interaction potential is represented as $M_{\lambda \mathbf{Q}}=C_{\lambda \mathbf{Q}} I\left(q_{z}\right)$, where $I\left(q_{z}\right)=\left|\left\langle 0\left|e^{i q_{z} z}\right| 0\right\rangle\right|^{2}$ is determined by the confinement potential which defines the ground state of $2 \mathrm{D}$ electrons, $|0\rangle$, and the function
$C_{\lambda \mathbf{Q}}$ is determined by both deformation-potential and piezoelectric mechanisms of interaction:

$$
\begin{array}{r}
C_{\lambda \mathbf{Q}}=\frac{1}{2 \rho_{M} \omega_{\lambda \mathbf{Q}}}\left[\mathcal{D}^{2} \sum_{i j} \mathrm{e}_{\lambda \mathbf{Q} i} \mathrm{e}_{\lambda \mathbf{Q} j} Q_{i} Q_{j}\right. \\
\left.+\frac{\left(e h_{14}\right)^{2}}{Q^{4}} \sum_{i j k, i^{\prime} j^{\prime} k^{\prime}} \kappa_{i j k} \kappa_{i^{\prime} j^{\prime} k^{\prime}} \mathrm{e}_{\lambda \mathbf{Q} k} \mathrm{e}_{\lambda \mathbf{Q} k^{\prime}} Q_{i} Q_{j} Q_{i^{\prime}} Q_{j^{\prime}}\right]
\end{array}
$$

Here $\mathcal{D}$ is the deformation potential constant, $h_{14}$ is the piezoelectric coupling constant, and $\rho_{M}$ is the material density. The sums are taken over Cartesian coordinate indices, $\mathrm{e}_{\lambda \mathbf{Q} i}$ are the components of the unit vector of the mode polarization, and the coefficient $\kappa_{i j k}$ is equal to unity if all the indices $i, j, k$ are different and equal to zero otherwise. The polarization vectors and the corresponding phonon mode frequencies are found from the eigenstate problem

$$
\sum_{j}\left[K_{i j}(\mathbf{Q})-\delta_{i j} \rho_{M} \Omega^{2}\right] \mathrm{e}_{\lambda \mathbf{Q} j}=0,
$$

where $K_{i j}(\mathbf{Q})$ is the dynamical matrix and $\delta_{i j}$ is the Kronecker symbol. For cubic crystals and in the elastic approximation, the dynamical matrix is expressed through three elastic constants which are written in the conventional notations:

$$
\begin{aligned}
K_{i j}(\mathbf{Q})= & {\left[\left(c_{11}-c_{44}\right) Q_{i}^{2}+c_{44} Q^{2}\right] \delta_{i j} } \\
& +\left(c_{12}+c_{44}\right) Q_{i} Q_{j}\left(1-\delta_{i j}\right) .
\end{aligned}
$$

The $2 \mathrm{D}$ vector $\mathbf{R}_{t_{1} t_{2}}$ standing in the exponential factors in Eqs. (3), (4), (7), and (8) includes both the term with dc field $\mathbf{E}=\left(E_{x}, E_{y}\right)$ and the $\mathrm{MW}$-induced term which depends on the frequency $\omega$ and amplitude $E_{\omega}$ of the MW field strength. It is convenient to use $\mathbf{R}^{ \pm}=R_{x} \pm i R_{y}$ given by

$$
\begin{array}{r}
R_{t_{1} t_{2}}^{+}=i \frac{e E_{+}\left(t_{1}-t_{2}\right)}{m \omega_{c}}-\frac{e E_{\omega}}{\sqrt{2} m \omega} \\
\times\left[s_{-}^{*}\left(e^{i \omega t_{1}}-e^{i \omega t_{2}}\right)+s_{+}\left(e^{-i \omega t_{1}}-e^{-i \omega t_{2}}\right)\right],
\end{array}
$$

and $R_{t_{1} t_{2}}^{-}=\left[R_{t_{1} t_{2}}^{+}\right]^{*}$, where $E_{ \pm}=E_{x} \pm i E_{y}$. The form of the complex coefficients $s_{ \pm}$depends on polarization of the radiation. In the case of linear polarization (used for the calculations below),

$$
s_{ \pm}=\frac{1}{\sqrt{2}} \frac{1}{\omega \pm \omega_{c}+i \omega_{p}}
$$

while in the case of circular $(-)$ polarization $s_{+}=0$ and $s_{-}=1 /\left(\omega-\omega_{c}+i \omega_{p}\right)$. Here $\omega_{p}$ is the radiative decay rate which determines the cyclotron line broadening because of electrodynamic screening effect. ${ }^{24,25,26}$ Under usual experimental conditions, when the electromagnetic radiation is normally incident from the vacuum on the sample with dielectric permittivity $\epsilon$, and $2 \mathrm{D}$ electron layer is close to the surface of the sample, one can find $\omega_{p}=2 \pi e^{2} n_{s} / m c \sqrt{\epsilon^{*}}$, where $\sqrt{\epsilon^{*}}=(1+\sqrt{\epsilon}) / 2$ and $n_{s}$ is 
the sheet electron density. It is assumed that $\omega_{p}$ is much larger than the transport scattering rate of electrons, this condition is amply satisfied for high-mobility electrons.

In contrast to $J^{i m}$ and $J^{p h}$ described above, the electron-electron collision integral $J^{e e}$ is not affected by the external fields. In Eqs. (5) and (9) the "exchange" Coulomb terms in the self-energies are neglected, since the main contribution to the electron-electron collision integral at low temperatures comes from small-angle scattering. The dynamical screening effects are also neglected. The statically-screened 2D interaction potential is written as

$$
U_{q}=\frac{2 \pi e^{2}}{\epsilon\left(q+q_{0}\right)},
$$

where $q_{0}=2 e^{2} m / \epsilon$ is the inverse screening length.

Having solved the kinetic equation (1), one can find the dissipative current density $\mathbf{j}$, averaged over the period $t_{\omega}=2 \pi / \omega$ of the microwaves:

$$
\mathbf{j}=\frac{2 e}{m} \int \frac{d \varepsilon}{2 \pi i} \int \frac{d \mathbf{p}}{(2 \pi)^{2}} \mathbf{p} \frac{1}{t_{\omega}} \int_{0}^{t_{\omega}} d t G_{\varepsilon \mathbf{p} t}^{-+} .
$$

The current can be also expressed through the distribution function $f_{\varepsilon \mathbf{p} t}$ with the aid of Eq. (10). The factor of 2 in Eq. (21) accounts for the spin degeneracy of electrons (the Zeeman splitting is neglected).

\section{CALCULATION OF RESISTIVITY}

It is assumed in the following that the temperature $T$ is small in comparison with the Fermi energy $\varepsilon_{F}$. Therefore, all the quantities whose dependence on the absolute value of electron momentum is slow can be taken at $p \equiv|\mathbf{p}|=p_{F}$, where $p_{F}$ is the Fermi momentum related to the sheet electron density $n_{s}$ as $p_{F}=\sqrt{2 \pi n_{s}}$. Accordingly, instead of the function $f_{\varepsilon \mathbf{p} t}$, one may use the distribution function $f_{\varepsilon \varphi t}$ which is equal to $f_{\varepsilon \mathbf{p} t}$ at $p=p_{F}$ and depends on the angle $\varphi$ of the quasiclassical momentum in the $2 \mathrm{D}$ plane. The absolute value of the momentum $\mathbf{q}=\mathbf{p}-\mathbf{p}^{\prime}$ transferred in the elastic scattering by impurities is then given by $q=2 p_{F} \sin (\theta / 2)$, where $\theta=\varphi-\varphi^{\prime}$ is the scattering angle. In a similar way, since the characteristic phonon energy $\omega_{\lambda \mathbf{Q}}$ is small compared to the Fermi energy, the electron-phonon scattering is treated in the quasielastic approximation, with $Q=\sqrt{q_{z}^{2}+4 p_{F}^{2} \sin ^{2}(\theta / 2)}$. The angle of the vector $\mathbf{q}$ is given by $\varphi_{q}=\pi / 2+\phi$, where $\phi=\left(\varphi+\varphi^{\prime}\right) / 2$.

Another approximation used below is the neglect of temporal harmonics of the distribution function $f_{\varepsilon \varphi t}$. Strictly speaking, excitation of such harmonics by microwaves can contribute to the dc current through the processes of second order in interaction, which leads to the so-called "photovoltaic" mechanism of MW photoresistance. ${ }^{9}$ According to theoretical estimates, this contribution is not large, and since no experimental evidence for the importance of the photovoltaic mechanism has been found, its neglect is justified. In the approximations described above, Eq. (1) is reduced to a stationary kinetic equation for time-independent distribution function $f_{\varepsilon \varphi}$ :

$$
\omega_{c} \frac{\partial f_{\varepsilon \varphi}}{\partial \varphi}=J_{\varepsilon \varphi}^{i m}+J_{\varepsilon \varphi}^{p h}+J_{\varepsilon \varphi}^{e e} .
$$

The collision integrals standing here are calculated by using the equations of Sec. II (for transformation of electron-impurity collision integral, see also Refs. 9 and 27). This leads to the following expressions:

$$
\begin{gathered}
J_{\varepsilon \varphi}^{i m}=\int_{0}^{2 \pi} \frac{d \varphi^{\prime}}{2 \pi} \nu(\theta) \sum_{n}\left[J_{n}(\beta)\right]^{2} \\
\times D_{\varepsilon+n \omega+\gamma}\left[f_{\varepsilon+n \omega+\gamma} \varphi^{\prime}-f_{\varepsilon \varphi}\right], \\
J_{\varepsilon \varphi}^{p h}=\int_{0}^{2 \pi} \frac{d \varphi^{\prime}}{2 \pi} \sum_{\lambda, n} \int_{0}^{\infty} \frac{d q_{z}}{\pi} m M_{\lambda \mathbf{Q}}\left[J_{n}(\beta)\right]^{2} \\
\times\left\{\left[\left(N_{\omega_{\lambda \mathbf{Q}}}+f_{\varepsilon \varphi}\right) f_{\varepsilon-\omega_{\lambda \mathbf{Q}}+n \omega+\gamma} \varphi^{\prime}\right.\right. \\
\left.\quad-\left(N_{\omega_{\lambda \mathbf{Q}}}+1\right) f_{\varepsilon \varphi}\right] D_{\varepsilon-\omega_{\lambda \mathbf{Q}}+n \omega+\gamma} \\
+\left[\left(N_{\omega_{\lambda \mathbf{Q}}}+1-f_{\varepsilon \varphi}\right) f_{\varepsilon+\omega_{\lambda \mathbf{Q}}+n \omega+\gamma} \varphi^{\prime}\right. \\
\left.\left.\quad-N_{\omega_{\lambda \mathbf{Q}}} f_{\varepsilon \varphi}\right] D_{\varepsilon+\omega_{\lambda \mathbf{Q}}+n \omega+\gamma}\right\} .
\end{gathered}
$$

The rate $\nu(\theta)$ characterizing the impurity scattering is defined as $\nu(\theta)=m w\left[2 p_{F} \sin (\theta / 2)\right]$, and the quantities $\omega_{\lambda \mathbf{Q}}$ and $M_{\lambda \mathbf{Q}}$ are expressed through the variables $q_{z}, \theta$, and $\phi$ as described above. Next, $J_{n}(\beta)$ is the Bessel function, and $D_{\varepsilon}=m^{-1} \int \frac{d \mathbf{p}}{(2 \pi)^{2}}\left(G_{\varepsilon \mathbf{p}}^{A}-G_{\varepsilon \mathbf{p}}^{R}\right) / i$ is the density of electron states expressed in units $m / \pi$ (so $D_{\varepsilon}=1$ at $B=0)$. In the presence of magnetic field $\left(\omega_{c} \ll \varepsilon_{F}\right.$ is assumed), the SCBA leads to the form

$$
\begin{gathered}
D_{\varepsilon}=1+2 \sum_{k=1}^{\infty} a_{k} \cos \frac{2 \pi k \varepsilon}{\omega_{c}}, \\
a_{k}=(-1)^{k} k^{-1} \exp \left(-\pi k / \omega_{c} \tau_{q}\right) L_{k-1}^{1}\left(2 \pi k / \omega_{c} \tau_{q}\right),
\end{gathered}
$$

where $\tau_{q}$ is the quantum lifetime of electrons and $L$ are the Laguerre polynomials. In the following, the case of overlapping Landau levels corresponding to weak magnetic fields is considered. The density of states is then given by the expression $D_{\varepsilon}=1-2 d \cos \left(2 \pi \varepsilon / \omega_{c}\right)$, where $d=\exp \left(-\pi / \omega_{c} \tau_{q}\right)$ is the Dingle factor, and $\tau_{q}$ is the quantum lifetime of electrons due to impurity scattering: $\tau_{q}^{-1}=\overline{\nu(\theta)}$ (here and below, the line over the function denotes the angular averaging $\left.(2 \pi)^{-1} \int_{0}^{2 \pi} d \theta \ldots\right)$. The angular-dependent variables $\beta$ and $\gamma$ standing in Eqs. (23) and (24) are proportional to the MW and dc fields, respectively:

$$
\beta=\frac{e E_{\omega} v_{F}}{\sqrt{2} \omega}\left|s_{-}\left(e^{i \varphi}-e^{i \varphi^{\prime}}\right)+s_{+}\left(e^{-i \varphi}-e^{-i \varphi^{\prime}}\right)\right|
$$

and

$$
\gamma=\frac{e v_{F}}{2 i \omega_{c}}\left[E_{-}\left(e^{i \varphi}-e^{i \varphi^{\prime}}\right)-E_{+}\left(e^{-i \varphi}-e^{-i \varphi^{\prime}}\right)\right],
$$


where $v_{F}=p_{F} / m$ is the Fermi velocity.

It is convenient to expand the distribution function in the angular harmonics: $f_{\varepsilon \varphi}=\sum_{k} f_{\varepsilon k} e^{i k \varphi}$. The density of dissipative electric current is determined by the $k=1$ harmonic. Applying the usual notation $j_{ \pm}=j_{x} \pm i j_{y}$, one has

$$
j_{-}=\frac{e p_{F}}{\pi} \int d \varepsilon D_{\varepsilon} f_{\varepsilon 1} .
$$

In the regime of classically strong magnetic fields, the anisotropic $(k \neq 0)$ part of the distribution function is expressed through the isotropic (angular-independent) part $f_{\varepsilon} \equiv f_{\varepsilon, k=0}$ directly from Eq. (22), by neglecting the angular dependence of all distribution functions in the collision integrals (23) and (24). The collision integral $J^{e e}$ does not lead to angular relaxation of the anisotropic distribution and, therefore, does not contribute to such a solution. A subsequent linearization of the collision integrals (23) and (24) with respect to the dc field $\mathbf{E}$ allows one to find a linear response to this field in Eq. (28). The response to $E_{-}$defines the symmetric part of the diagonal conductivity, $j_{-}=\sigma_{d} E_{-}$, where

$$
\begin{aligned}
\sigma_{d}= & -\frac{e^{2} n_{s}}{m \omega_{c}^{2}} \int_{0}^{2 \pi} \frac{d \theta}{2 \pi}(1-\cos \theta) \int_{0}^{2 \pi} \frac{d \phi}{2 \pi} \sum_{n}\left[J_{n}(\beta)\right]^{2} \\
& \times \int d \varepsilon\left[\nu(\theta) R_{\varepsilon n}^{i m}+\sum_{\lambda} \int_{0}^{\infty} \frac{d q_{z}}{\pi} 2 m M_{\lambda \mathbf{Q}} R_{\varepsilon n}^{p h}\right]
\end{aligned}
$$

with

$$
R_{\varepsilon n}^{i m}=D_{\varepsilon} D_{\varepsilon+n \omega} \frac{\partial f_{\varepsilon}}{\partial \varepsilon}+\left[f_{\varepsilon}-f_{\varepsilon+n \omega}\right] \frac{\partial D_{\varepsilon}}{\partial \varepsilon} D_{\varepsilon+n \omega}
$$

and

$$
\begin{array}{r}
R_{\varepsilon n}^{p h}=D_{\varepsilon} D_{\varepsilon-\omega_{\lambda \mathbf{Q}}+n \omega} \frac{\partial f_{\varepsilon}}{\partial \varepsilon}\left(N_{\omega_{\lambda \mathbf{Q}}}+1-f_{\varepsilon-\omega_{\lambda \mathbf{Q}}+n \omega}\right) \\
+\left[\left(N_{\omega_{\lambda \mathbf{Q}}}+1\right) f_{\varepsilon}-\left(N_{\omega_{\lambda \mathbf{Q}}}+f_{\varepsilon}\right) f_{\varepsilon-\omega_{\lambda \mathbf{Q}}+n \omega}\right] \\
\times \frac{\partial D_{\varepsilon}}{\partial \varepsilon} D_{\varepsilon-\omega_{\lambda \mathbf{Q}}+n \omega} .
\end{array}
$$

The resistivity $\rho$ is expressed as $\rho \simeq \sigma_{d} / \sigma_{\perp}^{2}$, where $\sigma_{\perp}=$ $e^{2} n_{s} / m \omega_{c}$ is the classical Hall conductivity.

In the absence of MW radiation, when $\beta=0$, one should substitute in Eq. (29) $\left[J_{n}(\beta)\right]^{2}=1$ for $n=0$, $\left[J_{n}(\beta)\right]^{2}=0$ for $n \neq 0$, and take into account that $f_{\varepsilon}$ is the equilibrium Fermi distribution function. Then, using $R_{\varepsilon 0}^{i m}=D_{\varepsilon}^{2}\left(\partial f_{\varepsilon} / \partial \varepsilon\right)$, and $R_{\varepsilon 0}^{p h}=-\left(N_{\omega_{\lambda \mathbf{Q}}}+1\right) f_{\varepsilon}(1-$ $\left.f_{\varepsilon-\omega_{\lambda \mathbf{Q}}}\right) D_{\varepsilon} D_{\varepsilon-\omega_{\lambda \mathrm{Q}}} / T$, one obtains the expression for the linear resistivity of $2 \mathrm{D}$ systems. The phonon-assisted contribution, which is proportional to the factor $R_{\varepsilon 0}^{p h}$, in this case is given by Eqs. (8) and (9) of Ref. 18.

The MW excitation leads to a significant modification of the resistivity. First, one should consider in Eq. (29) the terms with nonzero $n$, corresponding to absorption of $n$ quanta of radiation. Next, it is necessary to account for the changes of electron distribution function $f_{\varepsilon}$ owing to this absorption. This modified distribution function is found from the isotropic kinetic equation

$$
J_{\varepsilon}^{i m}+J_{\varepsilon}^{p h}+J_{\varepsilon}^{e e}=0 .
$$

The collision integrals $J_{\varepsilon}^{i m}$ and $J_{\varepsilon}^{p h}$ are given by Eqs. (23) and (24), where $\gamma=0$ and only the isotropic part of the distribution function is retained (since the anisotropic part is small, $f_{\varepsilon \varphi^{\prime}} \simeq f_{\varepsilon \varphi} \simeq f_{\varepsilon}$ ). The isotropic electronelectron collision integral is given by ${ }^{8,27}$

$$
\begin{aligned}
J_{\varepsilon}^{e e}= & \int d \varepsilon^{\prime} \int d \Omega A_{\varepsilon \varepsilon^{\prime}}(\Omega)\left[\left(1-f_{\varepsilon}\right)\left(1-f_{\varepsilon^{\prime}}\right) f_{\varepsilon+\Omega} f_{\varepsilon^{\prime}-\Omega}\right. \\
& \left.-f_{\varepsilon} f_{\varepsilon^{\prime}}\left(1-f_{\varepsilon+\Omega}\right)\left(1-f_{\varepsilon^{\prime}-\Omega}\right)\right] D_{\varepsilon^{\prime}} D_{\varepsilon+\Omega} D_{\varepsilon^{\prime}-\Omega}, \quad
\end{aligned}
$$

and the function $A_{\varepsilon \varepsilon^{\prime}}(\Omega)$ is estimated as $A \simeq$ $\left(2 \pi \varepsilon_{F}\right)^{-1} \ln \left(q_{0} / q_{1}\right)$, where $q_{1}$ is a characteristic (small) momentum separating the ballistic and diffusive regimes of electron-electron scattering; see Ref. 8 for a more detailed consideration. This energy-independent form of $A$ is valid in the experimentally relevant region of temperatures, where the transferred momentum $q \sim T / v_{F}$ lies in the interval $q_{0} \gg q \gg q_{1}$.

Below, only the terms linear in MW power are taken into account. Following the basic idea of Ref. 8, the distribution function is presented in the form

$$
f_{\varepsilon}=f_{\varepsilon}^{e}+\delta f_{\varepsilon}
$$

where $f_{\varepsilon}^{e}$ slowly varies with energy on the scale of $\omega_{c}$, and $\delta f_{\varepsilon}$ oscillates with the period $\omega_{c}$. The function $f_{\varepsilon}^{e}$ differs from the equilibrium Fermi distribution because of the influence of microwaves and satisfies Eq. (32) where the oscillating contribution to the density of states is neglected, $D_{\varepsilon}=1$.

One should notice that the electron-electron scattering is stronger than electron-phonon scattering and controls the electron distribution if the MW intensity is not high. Thus, the function $f_{\varepsilon}^{e}$ can be reasonably approximated by the heated Fermi distribution characterized by the electron temperature $T_{e}$. This temperature is determined from the balance equation

$$
W_{a}=W_{r}
$$

where $W_{a}$ is the MW power absorbed by the electron system and $W_{r}$ is the power transmitted out of this system through the energy relaxation owing to electron-phonon scattering. The balance equation can be obtained by integrating the kinetic equation (32), multiplied by the energy and density of states, over energy $\varepsilon$. This leads to the following expressions:

$$
W_{a}=\frac{m P_{\omega} \omega^{2}}{4 \pi}\left(\nu_{i m}^{t r}+\nu_{p h}^{a}(\omega)\right)
$$

where $\nu_{i m}^{t r}=\overline{\nu(\theta)(1-\cos \theta)}$ is the transport rate of electrons due to electron-impurity scattering and

$$
P_{\omega}=\left(\frac{e E_{\omega} v_{F}}{\hbar \omega}\right)^{2}\left(\left|s_{+}\right|^{2}+\left|s_{-}\right|^{2}\right)
$$


is the dimensionless function proportional to MW power. The rate

$$
\begin{array}{r}
\nu_{p h}^{a}(\omega)=\widehat{\mathcal{S}}_{1}\left\{2 N_{\omega_{\lambda \mathbf{Q}}}-N_{\omega_{\lambda \mathbf{Q}}-\omega}^{e}-N_{\omega_{\lambda \mathbf{Q}}+\omega}^{e}\right. \\
+2 \frac{\omega_{\lambda \mathbf{Q}}}{\omega}\left(N_{\omega_{\lambda \mathbf{Q}}-\omega}^{e}-N_{\omega_{\lambda \mathbf{Q}}+\omega}^{e}\right) \\
\left.+\frac{\omega_{\lambda \mathbf{Q}}^{2}}{\omega^{2}}\left(2 N_{\omega_{\lambda \mathbf{Q}}}^{e}-N_{\omega_{\lambda \mathbf{Q}}-\omega}^{e}-N_{\omega_{\lambda \mathbf{Q}}+\omega}^{e}\right)\right\}
\end{array}
$$

characterizes energy absorption due to electron-phonon scattering. The integral operators $\widehat{\mathcal{S}}_{n}$ are defined as

$$
\begin{aligned}
\widehat{\mathcal{S}}_{n}\{A\} \equiv \int_{0}^{2 \pi} & \frac{d \theta}{2 \pi} \int_{0}^{2 \pi} \frac{d \phi}{2 \pi} \sum_{\lambda} \int_{0}^{\infty} \frac{d q_{z}}{\pi} \\
& \times m M_{\lambda \mathbf{Q}}(1-\cos \theta)^{n} A,
\end{aligned}
$$

where $A$ is an arbitrary function which depends on the variables of integration. The function $N_{\Omega}^{e}=\left[e^{\Omega / T_{e}}-\right.$ 1] ${ }^{-1}$ differs from the Planck's distribution function $N_{\Omega}$ by substitution of electron temperature $T_{e}$ in place of the lattice temperature $T$. The introduction of the operators $\widehat{\mathcal{S}}_{n}$ considerably simplifies notations in the following. The power absorbed by the lattice is

$$
W_{r}=\frac{m}{\pi} \widehat{\mathcal{S}}_{0}\left\{\omega_{\lambda \mathbf{Q}}^{2}\left[N_{\omega_{\lambda \mathbf{Q}}}^{e}-N_{\omega_{\lambda \mathbf{Q}}}\right]\right\} .
$$

Assuming $T_{e} / T-1 \ll 1$, one gets $W_{r} \propto T_{e}-T$. According to the balance equation (35), this means that the heating is directly proportional to the MW power. Since only the contributions linear in power are considered, the relation $T_{e} / T-1 \ll 1$ must be satisfied. In this approximation, the balance equation is rewritten as

$$
\frac{T_{e}}{T}-1=P_{\omega}\left(\frac{\omega}{2 T}\right)^{2} \frac{\nu_{i m}^{t r}+\nu_{p h}^{a}(\omega)}{\nu_{p h}^{r}},
$$

where the energy relaxation rate $\nu_{p h}^{r}$ is introduced by the expression

$$
\nu_{p h}^{r}=\widehat{\mathcal{S}}_{0}\left\{\frac{\omega_{\lambda \mathbf{Q}}}{T} F\left(\frac{\omega_{\lambda \mathbf{Q}}}{2 T}\right)\right\},
$$

and $F(x)=[x / \sinh (x)]^{2}$. The function $F\left(\omega_{\lambda \mathbf{Q}} / 2 T\right)$ can be also expressed through the Planck's distribution, $F\left(\omega_{\lambda \mathbf{Q}} / 2 T\right)=\left(\omega_{\lambda \mathbf{Q}} / T\right)^{2} N_{\omega_{\lambda \mathbf{Q}}}\left(N_{\omega_{\lambda \mathbf{Q}}}+1\right)$. In the hightemperature limit, when $2 T_{e} \gg \omega, \omega_{\lambda \mathbf{Q}}$, the dependence $W_{r} \propto T_{e}-T$ takes place even at high MW power. In this limit, $\nu_{p h}^{a}(\omega)$ becomes frequency-independent and coincides with the transport rate due to electron-phonon scattering. This rate is defined as

$$
\nu_{p h}^{t r}=\widehat{\mathcal{S}}_{1}\left\{\frac{2 T}{\omega_{\lambda \mathbf{Q}}} F\left(\frac{\omega_{\lambda \mathbf{Q}}}{2 T}\right)\right\} .
$$

Once the distribution function $f_{\varepsilon}^{e}$ is known, one can find the rapidly oscillating correction $\delta f_{\varepsilon}$ from the oscillating (proportional to the Dingle factors) part of Eq.
(32). This leads to the following expression

$$
\begin{array}{r}
\frac{\delta f_{\varepsilon}}{\tau_{i n}}=\frac{P_{\omega}}{4} \mathcal{G}_{\omega} \nu_{i m}^{t r}+\widehat{\mathcal{S}}_{0}\left\{\mathcal{G}_{\omega_{\lambda \mathbf{Q}}}\left(N_{\omega_{\lambda \mathbf{Q}}}-N_{\omega_{\lambda \mathbf{Q}}}^{e}\right)\right\} \\
+\frac{P_{\omega}}{4} \widehat{\mathcal{S}}_{1}\left\{\mathcal{G}_{\omega-\omega_{\lambda \mathbf{Q}}}\left(N_{\omega_{\lambda \mathbf{Q}}}-N_{\omega_{\lambda \mathbf{Q}}-\omega}^{e}\right)+\mathcal{G}_{\omega+\omega_{\lambda \mathbf{Q}}}\right. \\
\left.\times\left(N_{\omega_{\lambda \mathbf{Q}}}-N_{\omega_{\lambda \mathbf{Q}}+\omega}^{e}\right)-2 \mathcal{G}_{\omega_{\lambda \mathbf{Q}}}\left(N_{\omega_{\lambda \mathbf{Q}}}-N_{\omega_{\lambda \mathbf{Q}}}^{e}\right)\right\},
\end{array}
$$

where

$$
\mathcal{G}_{\omega}=\delta D_{\varepsilon-\omega}\left(f_{\varepsilon-\omega}^{e}-f_{\varepsilon}^{e}\right)+\delta D_{\varepsilon+\omega}\left(f_{\varepsilon+\omega}^{e}-f_{\varepsilon}^{e}\right),
$$

and $\delta D_{\varepsilon}=D_{\varepsilon}-1=-2 d \cos \left(2 \pi \varepsilon / \omega_{c}\right)$. The inelastic scattering time $\tau_{i n}$, which describes relaxation of the isotropic part of the distribution function due to both electron-electron and electron-phonon scattering, is defined as $1 / \tau_{i n}=1 / \tau_{e e}+1 / \tau_{p h}$. The electron-electron scattering contribution has been widely discussed in the literature $^{8,27,28}$. The result for the electron-phonon scattering contribution is

$$
\frac{1}{\tau_{p h}} \simeq \widehat{\mathcal{S}}_{0}\left\{2 N_{\omega_{\lambda \mathbf{Q}}}+1+f_{\varepsilon+\omega_{\lambda \mathbf{Q}}}^{e}-f_{\varepsilon-\omega_{\lambda \mathbf{Q}}}^{e}\right\} .
$$

In derivation of this expression, the integrals $\widehat{\mathcal{S}}_{0}\left\{\left(2 N_{\omega_{\lambda \mathbf{Q}}}+1\right) \exp \left( \pm 2 \pi \omega_{\lambda \mathbf{Q}} / \omega_{c}\right)\right\}$ containing rapidly oscillating factors are neglected, which allows one to consider only the outscattering contribution in the collision integral. At high temperatures, $2 T>\omega_{\lambda \mathbf{Q}}$, energy dependence of $\tau_{p h}$ becomes inessential. For typical parameters of GaAs quantum wells, $1 / \tau_{p h}$ is small in comparison to $1 / \tau_{e e}$, so $\tau_{i n} \simeq \tau_{e e}$. According to Eq. (44), the function $\delta f_{\varepsilon}$ is composed from three terms: the first one is caused by electron-impurity scattering, while the two other terms are due to electron-phonon scattering. The second term does not oscillate with MW frequency and exists owing to electron heating. The third term is directly proportional to MW power and, in this sense, is analogous to the first term. Since only the effects linear in MW power are considered, the saturation $^{8,9}$ of $\delta f_{\varepsilon}$ is neglected. Strictly speaking, this approximation also requires a neglect of heating in the third term on the right-hand side of Eq. (44), because accounting for $T_{e} \neq T$ would produce the contributions of higher orders in MW power in this term.

Having found the distribution function, one can calculate the resistivity from Eqs. (29)-(31). It is written as

$$
\rho=\rho_{e}+\delta \rho,
$$

where $\rho_{e}$ and $\delta \rho$ are caused, respectively, by the components of the distribution function $f_{\varepsilon}^{e}$ and $\delta f_{\varepsilon}$. Consider first the modifications of resistivity associated with the function $f_{\varepsilon}^{e}$. The heated Fermi distribution $f_{\varepsilon}^{e}$ is substituted into Eqs. (30) and (31), and only the terms linear in MW power are retained $(n=0, \pm 1)$. The integral over $\varepsilon$ in Eq. (29) is calculated under the approximation

$$
2 \pi^{2} T \gg \omega_{c}
$$


when the Shubnikov-de Haas oscillations are thermally averaged out. The result of this integration can be divided into 5 parts:

$$
\rho_{e}=\rho_{i m}^{0}+\rho_{p h}^{0}+\rho_{i m}^{d i s}+\rho_{p h}^{c l}+\rho_{p h}^{d i s} .
$$

Two of these terms come from impurity-assisted (index $\mathrm{im}$ ) scattering, the first one is the resistivity without irradiation, and another one is a well-known ${ }^{8,9,27,28} \mathrm{MW}$ induced correction which describes MIRO due to displacement mechanism of photoresistance:

$$
\begin{gathered}
\rho_{i m}^{0}=\frac{m \nu_{i m}^{t r}}{e^{2} n_{s}}\left(1+2 d^{2}\right) \\
\rho_{i m}^{d i s}=-\frac{m \nu_{i m}^{*}}{e^{2} n_{s}} 2 d^{2} P_{\omega}\left[\sin ^{2} \frac{\pi \omega}{\omega_{c}}+\frac{\pi \omega}{\omega_{c}} \sin \frac{2 \pi \omega}{\omega_{c}}\right]
\end{gathered}
$$

where $\nu_{i m}^{*}=\overline{\nu(\theta)(1-\cos \theta)^{2}}$. This rate can be also expressed through the angular harmonics $\nu_{k}$ of the elastic scattering rate $\nu(\theta)$ as $\nu_{i m}^{*}=\left(3 \nu_{0}-4 \nu_{1}+\nu_{2}\right) / 2$.

The phonon-assisted (index $p h$ ) contribution comprises three terms. The first is described as the phonon-assisted resistivity modified by heating:

$$
\begin{array}{r}
\rho_{p h}^{0}=\frac{2 m}{e^{2} n_{s}} \widehat{\mathcal{S}}_{1}\left\{\mathcal{F}_{\lambda \mathbf{Q}}(0)\left(1+2 d^{2} \cos \frac{2 \pi \omega_{\lambda \mathbf{Q}}}{\omega_{c}}\right)\right. \\
\left.-2 d^{2} \mathcal{H}_{\lambda \mathbf{Q}}(0) \sin \frac{2 \pi \omega_{\lambda \mathbf{Q}}}{\omega_{c}}\right\} .
\end{array}
$$

Here and below,

$$
\begin{gathered}
\mathcal{F}_{\lambda \mathbf{Q}}(\omega)=N_{\omega_{\lambda \mathbf{Q}}}-N_{\omega_{\lambda \mathbf{Q}}+\omega}^{e} \\
+\frac{\omega_{\lambda \mathbf{Q}}+\omega}{T_{e}} N_{\omega_{\lambda \mathbf{Q}}+\omega}^{e}\left(N_{\omega_{\lambda \mathbf{Q}}+\omega}^{e}+1\right), \\
\mathcal{H}_{\lambda \mathbf{Q}}(\omega)=\left(N_{\omega_{\lambda \mathbf{Q}}}-N_{\omega_{\lambda \mathbf{Q}}+\omega}^{e}\right) \frac{2 \pi\left(\omega_{\lambda \mathbf{Q}}+\omega\right)}{\omega_{c}} .
\end{gathered}
$$

The term $\rho_{p h}^{0}$ does not oscillate as a function of $\omega$. In the limit $T_{e}=T$ it is reduced to the resistance $\rho_{p h}$ calculated in Ref. 18. The part of $\rho_{p h}^{0}$ proportional to the squared Dingle factor describes PIRO modified by heating (note that $\mathcal{H}_{\lambda \mathbf{Q}}(0)$ is nonzero only at $T_{e} \neq T$ ). The second phonon-assisted term is the MW-induced "classical" photoresistance, which is not related to quantum oscillations of the density of states and, therefore, does not oscillate with the magnetic field:

$$
\rho_{p h}^{c l}=\frac{m P_{\omega}}{2 e^{2} n_{s}} \widehat{\mathcal{S}}_{2}\left\{\mathcal{F}_{\lambda \mathbf{Q}}(\omega)+\mathcal{F}_{\lambda \mathbf{Q}}(-\omega)-2 \mathcal{F}_{\lambda \mathbf{Q}}(0)\right\} .
$$

This term essentially requires inelastic scattering: a formal limiting transition $\omega_{\lambda \mathbf{Q}} \rightarrow 0$ makes $\rho_{p h}^{c l}$ equal to zero. Therefore, $\rho_{p h}^{c l}$ has no analogue in the impurity-assisted tansport. The contribution (55) is strongly suppressed with decreasing frequency $\omega$ and increasing temperature
T. Finally, the third phonon-assisted term can be attributed to the displacement mechanism of MW photoresistance:

$$
\begin{array}{r}
\rho_{p h}^{d i s}=\frac{m}{e^{2} n_{s}} d^{2} P_{\omega} \widehat{\mathcal{S}}_{2}\left\{\mathcal{F}_{\lambda \mathbf{Q}}(-\omega) \cos \frac{2 \pi\left(\omega_{\lambda \mathbf{Q}}-\omega\right)}{\omega_{c}}\right. \\
+\mathcal{F}_{\lambda \mathbf{Q}}(\omega) \cos \frac{2 \pi\left(\omega_{\lambda \mathbf{Q}}+\omega\right)}{\omega_{c}}-2 \mathcal{F}_{\lambda \mathbf{Q}}(0) \cos \frac{2 \pi \omega_{\lambda \mathbf{Q}}}{\omega_{c}} \\
-\mathcal{H}_{\lambda \mathbf{Q}}(-\omega) \sin \frac{2 \pi\left(\omega_{\lambda \mathbf{Q}}-\omega\right)}{\omega_{c}}-\mathcal{H}_{\lambda \mathbf{Q}}(\omega) \\
\left.\times \sin \frac{2 \pi\left(\omega_{\lambda \mathbf{Q}}+\omega\right)}{\omega_{c}}+2 \mathcal{H}_{\lambda \mathbf{Q}}(0) \sin \frac{2 \pi \omega_{\lambda \mathbf{Q}}}{\omega_{c}}\right\} .
\end{array}
$$

In contrast to the impurity-assisted contribution $\rho_{i m}^{d i s}$, this term contains oscillating functions of $\left(\omega_{\lambda \mathbf{Q}} \pm \omega\right) / \omega_{c}$ describing interference of MIRO and PIRO.

The next step is to consider the terms associated with the oscillating part of the distribution function, $\delta f_{\varepsilon}$, generated by microwaves. Taking into account that $\delta f_{\varepsilon}$ is proportional to MW power, one should retain only the terms with $n=0$ in Eq. (29). As a result,

$$
\delta \rho=-\frac{m}{e^{2} n_{s}}\left[\nu_{i m}^{t r} \int d \varepsilon \delta R_{\varepsilon}^{i m}+2 \widehat{\mathcal{S}}_{1}\left\{\int d \varepsilon \delta R_{\varepsilon}^{p h}\right\}\right],
$$

where $\delta R_{\varepsilon}^{i m}$ and $\delta R_{\varepsilon}^{p h}$ are obtained by a linearization of $R_{\varepsilon 0}^{i m}$ and $R_{\varepsilon 0}^{p h}$ with respect to $\delta f_{\varepsilon}$ :

$$
\delta R_{\varepsilon}^{i m}=D_{\varepsilon}^{2} \frac{\partial \delta f_{\varepsilon}}{\partial \varepsilon}
$$

$$
\begin{array}{r}
\delta R_{\varepsilon}^{p h}=D_{\varepsilon} D_{\varepsilon-\omega_{\lambda \mathbf{Q}}} \frac{\partial \delta f_{\varepsilon}}{\partial \varepsilon}\left(N_{\omega_{\lambda \mathbf{Q}}}+1-f_{\varepsilon-\omega_{\lambda \mathbf{Q}}}^{e}\right) \\
-D_{\varepsilon} D_{\varepsilon-\omega_{\lambda \mathbf{Q}}} \frac{\partial f_{\varepsilon}^{e}}{\partial \varepsilon} \delta f_{\varepsilon-\omega_{\lambda \mathbf{Q}}}+\frac{\partial D_{\varepsilon}}{\partial \varepsilon} D_{\varepsilon-\omega_{\lambda \mathbf{Q}}} \\
\times\left[\left(N_{\omega_{\lambda \mathbf{Q}}}+1-f_{\varepsilon-\omega_{\lambda \mathbf{Q}}}^{e}\right) \delta f_{\varepsilon}\right. \\
\left.-\left(N_{\omega_{\lambda \mathbf{Q}}}+f_{\varepsilon}^{e}\right) \delta f_{\varepsilon-\omega_{\lambda \mathbf{Q}}}\right]
\end{array}
$$

The integration over energy in Eq. (57) is done with the aid of Eq. (44) for $\delta f_{\varepsilon}$ and under the approximation (48). The result for $\delta \rho$ appears to be rather complicated. It is presented below in a simplified form omitting the terms quadratic in electron-phonon scattering contributions:

$$
\begin{array}{r}
\delta \rho \simeq-\frac{m}{e^{2} n_{s}} 2 d^{2} \tau_{i n} \nu_{i m}^{t r}\left[P_{\omega} \nu_{i m}^{t r} \frac{2 \pi \omega}{\omega_{c}} \sin \frac{2 \pi \omega}{\omega_{c}}\right. \\
+4 \widehat{\mathcal{S}}_{0}\left\{\frac{2 \pi \omega_{\lambda \mathbf{Q}}}{\omega_{c}} \sin \frac{2 \pi \omega_{\lambda \mathbf{Q}}}{\omega_{c}}\left(N_{\omega_{\lambda \mathbf{Q}}}-N_{\omega_{\lambda \mathbf{Q}}}^{e}\right)\right\} \\
+2 P_{\omega} \widehat{\mathcal{S}}_{1}\left\{\mathcal{H}_{\lambda \mathbf{Q}}(-\omega) \sin \frac{2 \pi\left(\omega_{\lambda \mathbf{Q}}-\omega\right)}{\omega_{c}}\right. \\
+\mathcal{H}_{\lambda \mathbf{Q}}(\omega) \sin \frac{2 \pi\left(\omega_{\lambda \mathbf{Q}}+\omega\right)}{\omega_{c}}-2 \mathcal{H}_{\lambda \mathbf{Q}}(0) \sin \frac{2 \pi \omega_{\lambda \mathbf{Q}}}{\omega_{c}} \\
\times \cos ^{2} \frac{\pi \omega}{\omega_{c}}+\left(2 \mathcal{F}_{\lambda \mathbf{Q}}(0)-\mathcal{F}_{\lambda \mathbf{Q}}(-\omega)-\mathcal{F}_{\lambda \mathbf{Q}}(\omega)\right) \\
\times \cos \frac{2 \pi \omega}{\omega_{c}} \cos ^{2} \frac{\pi \omega_{\lambda \mathbf{Q}}}{\omega_{c}}+\frac{1}{2}\left(\mathcal{F}_{\lambda \mathbf{Q}}(\omega)-\mathcal{F}_{\lambda \mathbf{Q}}(-\omega)\right) \\
\left.\left.\times \sin \frac{2 \pi \omega}{\omega_{c}} \sin \frac{2 \pi \omega_{\lambda \mathbf{Q}}}{\omega_{c}}\right\}\right] .
\end{array}
$$


This form is valid under the condition $\nu_{i m}^{t r} \gg\left|\nu_{p h}^{c 1}\right|$ (see the definition of the rate $\nu_{p h}^{c 1}$ below and the corresponding discussion in the next section). The first term in the square brackets of Eq. (60) is the impurity-assisted scattering contribution. ${ }^{8}$ The second one is the phononassisted scattering contribution which is caused by heating and does not oscillate with MW frequency. The third one (proportional to $P_{\omega}$ ) is the phonon-assisted scattering contribution showing interference of MIRO and PIRO. Since only the terms linear in MW power are considered, the functions $\mathcal{F}$ and $\mathcal{H}$ in Eq. (60) should be taken at equilibrium, $T_{e}=T$. The same statement concerns Eq. (56).

The results are considerably simplified under the condition $2 T \gg \omega$. In this case one can write a general expression for the whole resistivity (47), which includes also the terms omitted previously in Eq. (60). It is convenient to represent the resistivity as a sum

$$
\rho=\rho_{b}+\rho_{o s},
$$

where the background (non-oscillating) part is

$$
\rho_{b}=\frac{m}{e^{2} n_{s}}\left[\nu_{i m}^{t r}\left(1+2 d^{2}\right)+\nu_{p h}^{t r}\right],
$$

and the oscillating part includes four terms:

$$
\begin{array}{r}
\rho_{o s}=\frac{m}{e^{2} n_{s}} 2 d^{2}\left\{\nu_{p h}^{c 1}\right. \\
-P_{\omega}\left(\nu_{i m}^{*}+\nu_{p h}^{c 2}\right)\left[\sin ^{2} \frac{\pi \omega}{\omega_{c}}+\frac{\pi \omega}{\omega_{c}} \sin \frac{2 \pi \omega}{\omega_{c}}\right] \\
-P_{\omega} \tau_{i n}\left(\nu_{i m}^{t r}+\nu_{p h}^{c 1}\right)^{2} \frac{2 \pi \omega}{\omega_{c}} \sin \frac{2 \pi \omega}{\omega_{c}} \\
\left.+\left(\frac{T_{e}}{T}-1\right)\left[\nu_{p h}^{s 1}+2 \nu_{p h}^{s 0} \tau_{i n}\left(\nu_{i m}^{t r}+\nu_{p h}^{c 1}\right)\right]\right\} .
\end{array}
$$

The phonon-assisted transport rate $\nu_{p h}^{t r}$ is given by Eq. (43), while the oscillating rates $\nu_{p h}^{c n}$ and $\nu_{p h}^{s n}$ are defined as

$$
\begin{aligned}
& \nu_{p h}^{c n}=\widehat{\mathcal{S}}_{n}\left\{\frac{2 T}{\omega_{\lambda \mathbf{Q}}} \cos \frac{2 \pi \omega_{\lambda \mathbf{Q}}}{\omega_{c}} F\left(\frac{\omega_{\lambda \mathbf{Q}}}{2 T}\right)\right\}, \\
& \nu_{p h}^{s n}=\frac{4 \pi T}{\omega_{c}} \widehat{\mathcal{S}}_{n}\left\{\sin \frac{2 \pi \omega_{\lambda \mathbf{Q}}}{\omega_{c}} F\left(\frac{\omega_{\lambda \mathbf{Q}}}{2 T}\right)\right\} .
\end{aligned}
$$

As seen from this definition, oscillations of $\nu_{p h}^{c n}$ and $\nu_{p h}^{s n}$ with the magnetic field have different phases governed by cosine (index $c$ ) and sine (index $s$ ) functions, respectively. The rate $\nu_{p h}^{c 1}$ differs from $\nu_{p h}^{t r}$ by the presence of the oscillating factor $\cos \left(2 \pi \omega_{\lambda \mathbf{Q}} / \omega_{c}\right)$ under the integral operator. This rate describes equilibrium PIRO, the first term in Eq. (63), in the approximation of overlapping Landau levels $\left(d^{2} \ll 1\right)$ used here (notice that in Ref. $18 \nu_{p h}^{c 1}$ is denoted as $\left.\nu_{p h}^{(1)}\right)$. The direct influence of microwaves on magnetoresistance is described by the second and the third terms in Eq. (63), which are proportional to $P_{\omega}$. These terms express contributions of the displacement and inelastic mechanisms of photoresistance, respectively. Their frequency dependence is given by the same oscillating functions as in the case of impurity-assisted transport, $, 9,27,28$ while the scattering rates are modified by electron-phonon interaction: $\nu_{i m}^{*} \rightarrow \nu_{i m}^{*}+\nu_{p h}^{c 2}$ and $\nu_{i m}^{t r} \rightarrow \nu_{i m}^{t r}+\nu_{p h}^{c 1}$. This modification is very essential because the rates $\nu_{p h}^{c 2}$ and $\nu_{p h}^{c 1}$ oscillate with the magnetic field. The interference of phonon-induced magnetoresistance oscillations with MIRO is produced as a result of multiplication of these rates by the frequency-dependent oscillating functions. Finally, the last term in Eq. (63) has no analogue in the theory of impurity-assisted magnetotransport. It is proportional to $T_{e}-T$ and can be described as a result of MW heating on phonon-assisted magnetoresistance. This term does not oscillate with frequency $\omega$ but it oscillates with the magnetic field, similar to the equilibrium magnetoresistance. The phase of these oscillations is shifted with respect to the phase of equilibrium PIRO. Notice that the heating factor $T_{e} / T-1$ is expressed through $P_{\omega}$ with the aid of Eq. (41), so the whole MW-induced correction to $\rho$ is proportional to $P_{\omega}$.

\section{NUMERICAL RESULTS AND DISCUSSION}

As follows from the above consideration, quantum oscillations of resistivity in 2D electron layers irradiated by microwaves demonstrate a rich and complicated behavior if electron-phonon interaction is taken into account. The absorption of microwaves in the presence of this interaction leads to the appearance of various additional oscillating contributions to magnetoresistance, Eq. (63). These contributions are similar to equilibrium PIRO in the sense that their periodicity depends on characteristic phonon frequencies $\omega_{\lambda \mathbf{Q}}$ with $Q=2 p_{F}$. However, they are essentially non-equilibrium and some of them (those entering the last term of Eq. (63)) have a phase different from that of equilibrium PIRO. The non-equilibrium phonon-induced contributions entering the second and the third term of Eq. (63) show the periodicity determined by combined frequencies such as $\omega \pm \omega_{\lambda \mathbf{Q}}$ and correspond to the interference of PIRO and MIRO.

To illustrate the influence of microwaves on magnetoresistance and to compare contributions of different terms in Eq. (63), numerical calculations have been carried out. The sample parameters are taken for [001]-grown GaAs quantum wells of high mobility $\left(1.17 \times 10^{7} \mathrm{~cm}^{2} / \mathrm{V} \mathrm{s}\right.$ at low temperature) experimentally investigated in Ref. 17 without MW excitation. The list of parameters necessary for description of electron-phonon interaction can be found in Ref. 18. The inelastic scattering time was estimated according to the relation ${ }^{8}$

$$
\frac{1}{\tau_{i n}} \simeq \lambda_{i n} \frac{T^{2}}{\varepsilon_{F}}
$$


where $\lambda_{i n}$ is a numerical constant of order unity. For calculations, $\lambda_{i n}=1$ was chosen, which is consistent with experimental data. ${ }^{29}$ The rate $\nu_{i m}^{*}$ characterizing the displacement mechanism of photoresistance under impurityassisted scattering has been calculated with the aid of the model of long-range random impurity potential described by the correlator $w(q) \propto \exp \left(-l_{c} q\right)$. The correlation length, $l_{c} \gg p_{F}^{-1}$, is determined by using the transport rate $\nu_{i m}^{t r}$ found from the given mobility and quantum lifetime $\tau_{q}=15$ ps estimated in Ref. 17.
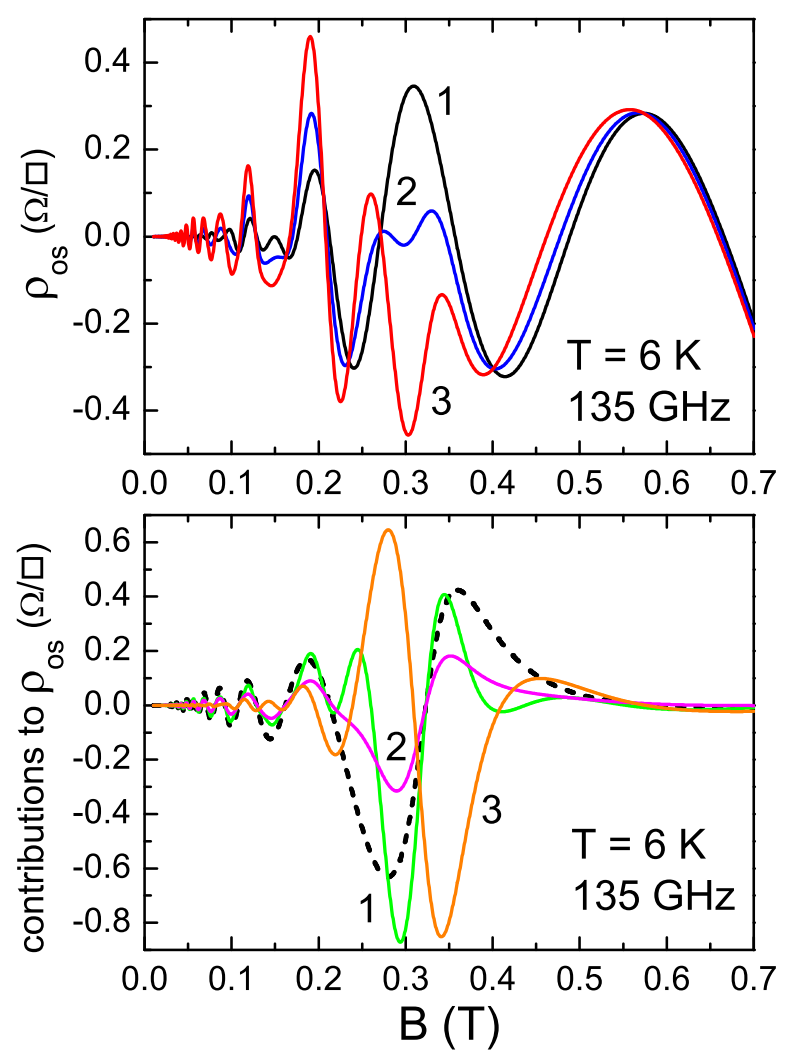

FIG. 1: (Color online) Upper panel. Magnetic-field dependence of the oscillating part of resistivity, $\rho_{o s}$, calculated for the quantum well of Ref. 17 at $T=6 \mathrm{~K}$ in equilibrium (black, curve 1) and under $135 \mathrm{GHz} \mathrm{MW}$ excitation with $E_{\omega}=2$ $\mathrm{V} / \mathrm{cm}$ (blue, curve 2) and $E_{\omega}=3 \mathrm{~V} / \mathrm{cm}$ (red, curve 3 ). Lower panel. MW-induced contributions to $\rho_{\text {os }}$ at $E_{\omega}=3 \mathrm{~V} / \mathrm{cm}$ : displacement mechanism, second term in Eq. 63 (green, curve 1), inelastic mechanism, third term in Eq. 63 (magenta, curve 2), non-equilibrium PIRO, fourth term in Eq. 63 (orange, curve 3 ). The contribution due to elastic impurity scattering (MIRO) is shown by black dashed line.

Figure 1 shows the oscillating part of resistivity at a lattice temperature of $6 \mathrm{~K}$ without microwaves (in equilibrium) and under $135 \mathrm{GHz}$ excitation whose intensity is determined by the MW electric field $E_{\omega}$. For the chosen frequency, the increase in intensity leads to en- hancement of PIRO maxima at 0.12 and $0.18 \mathrm{~T}$, while the maximum at $0.3 \mathrm{~T}$ is suppressed and eventually inverted, and two additional maxima appear at its sides. The inversion of this PIRO peak cannot be explained as a result of simple superposition of phonon-induced and MW-induced oscillations and is attributed to interference of these kinds of oscillations, as reflected in Eq. (63). The lower panel of Fig. 1 shows relative contributions to the oscillating resistivity. Apart from the impurity-induced contribution (MIRO), this plot shows the total contribution of displacement mechanism, inelastic mechanism, and non-equilibrium PIRO. All these contributions are comparable, though the inelastic mechanism contribution is smaller than the others in the region around $0.3 \mathrm{~T}$. The $B$-dependence of this contribution closely follows the MIRO plot, showing slight deviations caused by phononinduced oscillations. In contrast, such deviations are very pronounced in the displacement mechanism contribution (see, for example, peak at $0.25 \mathrm{~T}$ and minimum at 0.4 $\mathrm{T})$. The contribution of non-equilibrium PIRO is clearly shifted by phase with respect to the other contributions. This shift leads to a partial compensation of different contributions, which reduces the influence of microwaves on magnetoresistance.

The effect of microwaves of a smaller frequency, 50 $\mathrm{GHz}$, is illustrated in Fig. 2. The magnetoresistance is considerably changed only in the low-field region. These changes are similar to those in Fig. 1: PIRO peaks are either enhanced or inverted by microwaves. Again, the inelastic mechanism contribution is weakly modified by electron-phonon interaction, while the displacement mechanism contribution is strongly modified by this interaction and shows numerous oscillations related to interference of PIRO with MIRO. The non-equilibrium PIRO contribution is smaller than the others. The relative decrease of this contribution with lowering frequency also follows from Eqs. (63) and (41).

The calculations demonstrate that the displacement mechanism of MW photoresistance is much stronger influenced by electron-phonon interaction than the inelastic mechanism. This property is understood from Eq. (63) showing that the relative contributions caused by phonons for these two mechanisms are determined by the ratios $\left|\nu_{p h}^{c 2}\right| / \nu_{i m}^{*}$ and $\left|\nu_{p h}^{c 1}\right| / \nu_{i m}^{t r}$, respectively. At $T=6 \mathrm{~K}$ the second ratio is small, as also seen from experimental data: ${ }^{17}$ the amplitudes of equilibrium PIRO governed by the rate $\nu_{p h}^{c 1}$ are small compared to the background resistivity. On the other hand, for long-range impurity potentials one has $\nu_{i m}^{*} \ll \nu_{i m}^{t r}$, while $\nu_{p h}^{c 2}$ is approximately twice larger by amplitude than $\nu_{p h}^{c 1}$, since the main contribution to the oscillating phonon-assisted rates $\nu_{p h}^{c n}$ comes from backscattering processes, $\theta \simeq \pi$. Therefore, one always has

$$
\frac{\left|\nu_{p h}^{c 2}\right|}{\nu_{i m}^{*}} \gg \frac{\left|\nu_{p h}^{c 1}\right|}{\nu_{i m}^{t r}} .
$$

With increasing temperature, when electron-phonon scattering gets stronger and the rates $\nu_{p h}^{c n}$ increase, the 

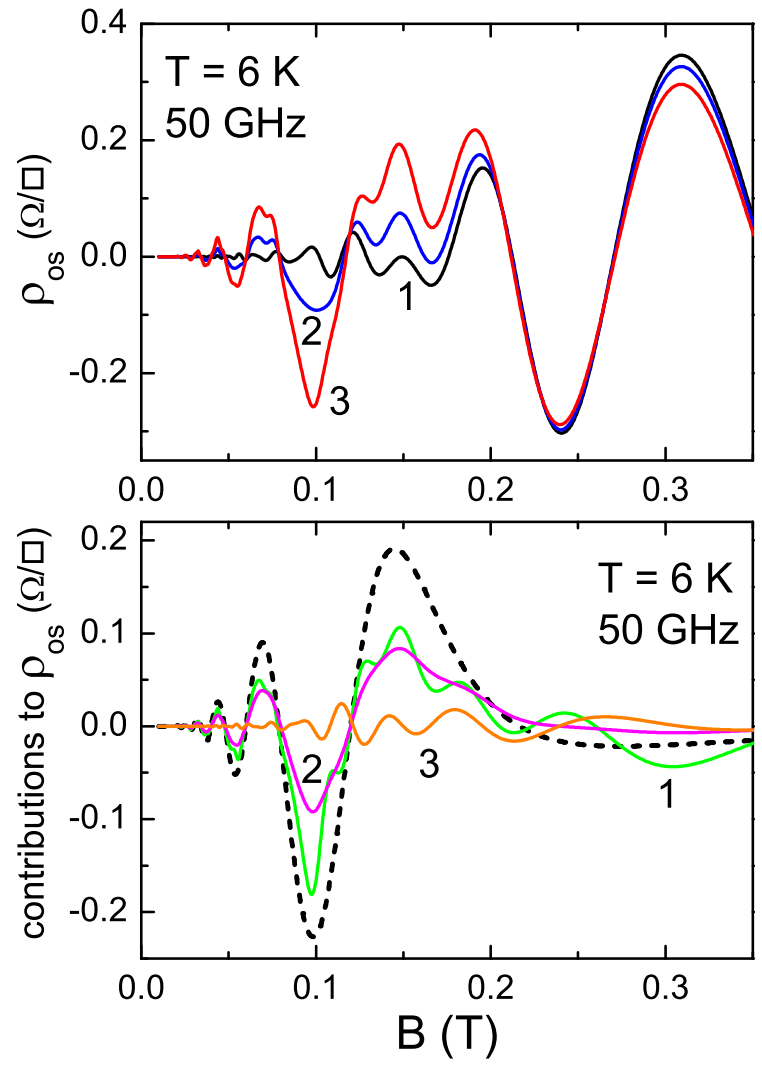

FIG. 2: (Color online) The same as in Fig. 1 for $50 \mathrm{GHz}$ MW excitation with $E_{\omega}=0.5 \mathrm{~V} / \mathrm{cm}$ (blue, curve 2) and $E_{\omega}=0.8$ $\mathrm{V} / \mathrm{cm}$ (red, curve 3). The MW-induced contributions in the lower panel are shown for $E_{\omega}=0.8 \mathrm{~V} / \mathrm{cm}$.

ratio $\left|\nu_{p h}^{c 2}\right| / \nu_{i m}^{*}$ becomes comparable to unity and the displacement mechanism contribution is considerably modified by this scattering. This corresponds to the temperature chosen for the calculations. Further increase in temperature leads to an interesting situation when the amplitudes of $\nu_{p h}^{c 2}$ become much larger than $\nu_{i m}^{*}$, so the displacement mechanism of MW photoresistance is dominated by electron-phonon scattering.

It is worth noting that at low temperatures $(T \simeq 1$ $\mathrm{K}$ ), when phonon-assisted contributions are yet frozen out, the inelastic mechanism is parametrically stronger 8,9 than the displacement one, because of large value of $\tau_{i n}$. However, in the samples of very high mobility, like those investigated in Ref. 17, the contribution of the displacement mechanism experimentally proves to be important ${ }^{12}$ starting from $T=2 \mathrm{~K}$. According to estimates, at this temperature the product $\tau_{i n} \nu_{i m}^{t r}$ determining the strength of inelastic mechanism is smaller than unity. As follows from Eq. (63), the parameter $\tau_{i n} \nu_{i m}^{t r}$ also determines the relative strength of phononassisted contribution in the inelastic (third) term compared to the similar contribution in the displacement (second) term. Therefore, in the temperature region when phonon-induced oscillating contributions to resistivity become thermally activated $(T>2 \mathrm{~K})$, their relative influence on the MW photoresistance via inelastic mechanism is already suppressed. In particular, at $T=6$ $\mathrm{K}$ a strong inequality,

$$
\tau_{i n} \nu_{i m}^{t r} \ll 1,
$$

allows one to neglect the phonon-induced oscillating photoresistance due to inelastic mechanism. For the samples of moderate mobility $\left(\sim 10^{6} \mathrm{~cm}^{2} / \mathrm{V} \mathrm{s}\right)$, the applicability of Eq. (68) requires higher temperatures. Nevertheless, in the low-temperature region $\left(\tau_{i n} \nu_{i m}^{t r}>1\right)$ the ratio $\left|\nu_{p h}^{c 1}\right| / \nu_{i m}^{t r}$ remains small, and phonon-induced photoresistance due to inelastic mechanism can be neglected in comparison with the impurity-induced photoresistance. Notice also that the condition (68), being applied to nonequilibrium PIRO (the last term in Eq. (63)), allows one to neglect the part which comes from $\delta \rho$ and is proportional to $\tau_{i n}$. Thus, under condition (68) one may always ignore the contribution to $\delta \rho$ caused by electron-phonon interaction. In contrast, the non-equilibrium contributions to $\rho_{e}$ caused by this interaction cannot be ignored and are important for evaluation of MW-induced magnetoresistance in 2D systems where PIRO is observed. These contributions are described by the oscillating rates $\nu_{p h}^{c 2}$ and $\nu_{p h}^{s 1}$ in Eq. (63).

The main approximations used in the paper are discussed below in more detail.

The approximation of overlapping Landau levels corresponds to a simplified (single-mode) description of the oscillating density of electron states in contrast to the exact SCBA expression (25). Though in high-mobility samples separation of Landau levels becomes essential already at $B \simeq 0.1 \mathrm{~T}$, this description produces a very good agreement with the calculation based on Eq. (25) (see Ref. 18) for equilibrium oscillating magnetoresistance up to $B=0.4 \mathrm{~T}$. This means that the first PIRO harmonic dominates in the main interval of magnetic fields under consideration, and only the last PIRO peak at $B \simeq 0.6$ $\mathrm{T}$ appears to be slightly sensitive to the shape of the density of states. Besides, regarding a limited validity of the SCBA in the regime of separated Landau levels, the single-mode description proves to be a reasonable choice. It also provides a rigorous justification for introduction of inelastic scattering time necessary for analytical description of inelastic mechanism of photoresistance.

The assumption of weak MW excitation means that only linear terms in the expansion of magnetoresistance in powers of MW intensity are taken into account. First, this implies a neglect of multi-photon absorption processes and formally requires that the argument $\beta$ of the Bessel function in Eq. (29) must be small for the scattering angles essential in the integration. Since the phononassisted scattering occurs in a broad range of angles, the condition $\beta \ll 1$ is equivalent to $P_{\omega} \ll 1$ and is satisfied by a proper choice of the MW field strength $E_{\omega}$. Next, the neglect of saturation effect for inelastic mechanism 
contribution $^{8,9}$ requires $\tau_{i n} \nu_{i m}^{t r} P_{\omega} \ll 1$. As shown above, the phonon-induced photoresistance oscillations in highmobility layers are essential at $\tau_{i n} \nu_{i m}^{t r}<1$, so the neglect of saturation is also satisfied at $P_{\omega} \ll 1$. Finally, the absence of strong heating, $T_{e} / T-1 \ll 1$, is equivalent to smallness of the right-hand side of Eq. (41) and also can be expressed in terms of smallness of $E_{\omega}$.

Coming back to multi-photon processes, it is worth noting that phonon-assisted multi-photon absorption requires much weaker MW fields than impurity-assisted multi-photon absorption. The reason is the longrange nature of impurity potential in high-mobility (modulation-doped) structures, which leads to smallangle scattering $(\theta \ll 1)$ and, thereby, effectively smaller $\beta$ in the impurity term of Eq. (29). Since the multiphoton processes are thought to be responsible for the phenomenon of fractional MIRO observed at elevated MW intensities, ${ }^{10,30-35}$ the above conclusion may be useful for interpretation of relevant experimental data. In particular, a recent experiment ${ }^{34}$ shows several highorder fractional resonances in photoresistance when the MW power is still insufficient to produce these features as a result of multi-photon processes under electronimpurity scattering. A consideration of electron-phonon interaction, which enables multi-photon processes at weaker MW power, could possibly explain the data of Ref. 34 (indeed, the high-order fractional features are observed at $T=6.5 \mathrm{~K}$, when phonon-assisted scattering may become important). A more detailed consideration of this problem requires a separate study which is beyond the scope of the present paper.

The neglect of electron-phonon and electron-electron interaction in calculation of the Green's function in Eq. (14) is justified at low temperatures, when the inverse quantum lifetime due to impurity scattering, $1 / \tau_{q}$, is much larger than the corresponding inelastic scattering rates. This leads to temperature-independent density of states characterized by the Dingle factors used in the above calculations. However, while electron-phonon contribution is not essential in a wide temperature range, the contribution of electron-electron scattering to the Landau level broadening cannot be disregarded already at several Kelvin. To take this effect into account, one should replace the Dingle factors $d$ with temperature-dependent functions $d(T)=\exp \left[-\pi / \omega_{c} \tau_{q}(T)\right]$, where

$$
\frac{1}{\tau_{q}(T)}=\frac{1}{\tau_{q}}+\frac{1}{\tau_{q}^{e e}}, \quad \frac{1}{\tau_{q}^{e e}} \simeq \lambda \frac{T^{2}}{\varepsilon_{F}}
$$

and the numerical constant $\lambda$ (usually determined experimentally) is of the order of unity. The reliability of this approach is justified theoretically and proved in numerous works by studying temperature dependence of quantum oscillations in magnetoresistance. ${ }^{12,17,28,36,37,38,39}$ The inclusion of temperature-dependent Dingle factors into Eq. (63) leads, in particular, to non-monotonic temperature dependence of PIRO amplitudes, which is also observed in experiments. ${ }^{17,40}$
The consideration was done for the case of spatially homogeneous 2D electron system. The effects of a finite size of the sample, in particular, the influence of contacts and edges on the MW photoresistance, have been neglected. This includes, for instance, the neglect of confined magnetoplasmon effects in MW absorption. ${ }^{41}$ The problem of magnetoresistance under inhomogeneous MW field distribution appears to be even more important. The observed insensitivity of MIRO to the sense of circular polarization, ${ }^{42}$ the absence of MIRO in contactless measurenents, ${ }^{43}$ and calculations of the field distribution in $2 \mathrm{D}$ systems with metallic contacts ${ }^{44}$ suggest possible near-contact origin of MIRO phenomenon in highmobility layers. This means that, owing to large MW field gradients in the vicinity of contacts, there may exist an additional (caused by near-contact regions) oscillating contribution to resistivity ${ }^{44}$ which exceeds the contribution caused by uniform MW field in the bulk of the layer. The nature of the assumed near-contact oscillating contribution remains unclear, and its theoretical description is currently missing. Further investigation of this problem is necessary. In the meanwhile, any extension of theoretical knowledge about MW-induced response based on bulk properties of $2 \mathrm{D}$ electron gas is important and may help to uncover the exact nature of MIRO.

In summary, a theoretical study of the effects of continuous MW irradiation on the magnetoresistance of 2D electrons interacting with impurities and acoustic phonons is presented. The main subject of the study was the quantum oscillations of resistivity caused by the scattering-assisted electron transitions between different Landau levels. In the absence of microwaves (in equilibrium), there is only one kind of such oscillations (PIRO) owing to electron-phonon scattering. When MW radiation is applied, the transitions between Landau levels can occur with absorption or emission of MW quanta, either under elastic scattering by impurities or under inelastic scattering by acoustic phonons. The concept of microscopic mechanisms of MW photoresistance (displacement and inelastic mechanisms), previously developed for the case of electrons interacting with impurities, applies to both these kinds of transitions. The impurity-assisted transitions are responsible for the oscillations (MIRO) whose $1 / B$ periodicity is determined solely by the MW frequency $\omega$. The phonon-assisted transitions lead to more complicated oscillations which are described as a result of PIRO-MIRO interference. It is demonstrated that such oscillations are attributed mostly to the displacement mechanism. Finally, heating of electron system by microwaves gives rise to an additional phonon-induced oscillating contribution, denoted here as non-equilibrium PIRO, which is shifted by phase with respect to equilibrium PIRO. This contribution is not related to specific features of MW excitation and appears also when electron system is heated by a dc field. Accounting for all the oscillating contributions leads to a peculiar magnetoresistance picture that should be observable in highmobility 2D layers if the temperature is high enough so 
the electron-phonon scattering is not frozen out. Some relevant examples are given in Figs. 1 and 2 .

One of the primary goals of this work was to emphasize the importance of electron-phonon interaction in description of the microwave-induced oscillating magnetoresistance. The author believes that the results and conclusions of this research will be useful for better understanding of existing experimental data and may stimulate further experiments and theoretical studies.

Acknowledgement: The author is grateful to I. A. Dmitriev for a helpful discussion of the method of moving coordinate frame.
[1] M. A. Zudov, R. R. Du, J. A. Simmons, and J. L. Reno, Phys. Rev. B 64, 201311(R) (2001).

[2] R. G. Mani, J. H. Smet, K. von Klitzing, V. Narayanamurti, W. B. Johnson, and V. Umansky, Nature 420, 646 (2002).

[3] M. A. Zudov, R. R. Du, L. N. Pfeiffer, and K. W. West, Phys. Rev. Lett. 90, 046807 (2003).

[4] R. L. Willett, L. N. Pfeiffer, and K. W. West, Phys. Rev. Lett. 93, 026804 (2004).

[5] V. I. Ryzhii, Sov. Phys. Solid State 11, 2078 (1970); V. I. Ryzhii, R. A. Suris, and B.S. Shchamkhalova, Sov. Phys. Semicond. 20, 1299 (1986).

[6] A. C. Durst, S. Sachdev, N. Read, and S. M. Girvin, Phys. Rev. Lett 91, 086803 (2003).

[7] M. G. Vavilov and I. L. Aleiner, Phys. Rev. B 69, 035303 (2004).

[8] I. A. Dmitriev, M. G. Vavilov, I. L. Aleiner, A. D. Mirlin, and D. G. Polyakov, Phys. Rev. B 71, 115316 (2005).

[9] I. A. Dmitriev, A. D. Mirlin, and D. G. Polyakov, Phys. Rev. B 75, 245320 (2007).

[10] S. I. Dorozhkin, J. H. Smet, K. von Klitzing, L. N. Pfeiffer, and K. W. West, JETP Lett. 86, 543 (2007).

[11] S. Wiedmann, G. M. Gusev, O.E. Raichev, T. E. Lamas, A. K. Bakarov, and J. C. Portal, Phys. Rev. B 78, 121301(R) (2008).

[12] A. T. Hatke, M. A. Zudov, L. N. Pfeiffer, and K. W. West, Phys. Rev. Lett. 102, 066804 (2009).

[13] M. A. Zudov, I.V. Ponomarev, A. L. Efros, R. R. Du, J. A. Simmons, and J. L. Reno, Phys. Rev. Lett. 86, 3614 (2001).

[14] J. Zhang, S. K. Lyo, R. R. Du, J. A. Simmons, and J. L. Reno, Phys. Rev. Lett. 92, 156802 (2004).

[15] A. A. Bykov, A. K. Kalagin and A. K. Bakarov, JETP Lett. 81, 523 (2005).

[16] W. Zhang, M. A. Zudov, L. N. Pfeiffer, and K. W. West, Phys. Rev. Lett. 100, 036805 (2008).

[17] A. T. Hatke, M. A. Zudov, L. N. Pfeiffer, and K. W. West, Phys. Rev. Lett. 102, 086808 (2009).

[18] O. E. Raichev, Phys. Rev. B, 80, 075318 (2009).

[19] Y. Ma, R. Fletcher, E. Zaremba, M. D'Iorio, C. T. Foxon, and J. J. Harris, Phys. Rev. B 43, 9033 (1991).

[20] X. L. Lei and S. Y. Liu, Phys. Rev. B 72, 075345 (2005).

[21] J. Inarrea and G. Platero, Phys. Rev. B 72, 193414 (2005).

[22] X. L. Lei, J. Phys.: Condens. Matter 16, 4045 (2004).

[23] V. Ryzhii and V. Vyurkov, Phys. Rev. B 68, 165406 (2003); V. Ryzhii, arxiv:cond-mat/0305484.

[24] K. W. Chiu, T. K. Lee, and J. J. Quinn, Surf. Sci. 58, 182 (1976).

[25] S. A. Mikhailov, Phys. Rev. B 70, 165311 (2004).
[26] S. A. Studenikin, M. Potemski, A. Sachrajda, M. Hilke, L. N. Pfeiffer, and K. W. West, Phys. Rev. B 71, 245313 (2005).

[27] M. Khodas and M. G. Vavilov, Phys. Rev. B 78, 245319 (2008).

[28] I.A. Dmitriev, M. Khodas, A.D. Mirlin, D.G. Polyakov, M.G. Vavilov, Phys. Rev. B 80, 165327 (2009).

[29] S. Wiedmann, G. M. Gusev, O. E. Raichev, A. K. Bakarov, and J. C. Portal, Phys. Rev. B 81, 085311 (2010), and references therein.

[30] R.G. Mani, J. H. Smet, K. von Klitzing, V. Narayanamurti, W. B. Johnson, and V. Umansky, Phys. Rev. Lett. 92, 146801 (2004).

[31] S. I. Dorozhkin, J. H. Smet, V. Umansky, and K. von Klitzing, Phys. Rev. B 71, 201306(R) (2005).

[32] M. A. Zudov, R. R. Du, L. N. Pfeiffer, and K. W. West, Phys. Rev. B 73, 041303(R) (2006).

[33] I. A. Dmitriev, A. D. Mirlin, and D. G. Polyakov, Phys. Rev. Lett. 99, 206805 (2007).

[34] S. Wiedmann, G. M. Gusev, O. E. Raichev, A. K. Bakarov, and J. C. Portal, Phys. Rev. B, 80, 035317 (2009).

[35] M. Khodas, H.-S. Chiang, A. T. Hatke, M. A. Zudov, M. G. Vavilov, L. N. Pfeiffer, and K. W. West, arxiv:condmat/0912.1364.

[36] N. C. Mamani, G. M. Gusev, T. E. Lamas, A. K. Bakarov, and O. E. Raichev, Phys. Rev. B 77, 205327 (2008).

[37] A. V. Goran, A. A. Bykov, A. I. Toropov, and S. A. Vitkalov, Phys. Rev. B 80, 193305 (2009).

[38] S. Wiedmann, N. C. Mamani, G. M. Gusev, O. E. Raichev, A. K. Bakarov, and J. C. Portal, Phys. Rev. B 80, 245306 (2009).

[39] A. T. Hatke, M. A. Zudov, L. N. Pfeiffer, and K. W. West, Phys. Rev. B 79, 161308(R) (2009).

[40] A. A. Bykov and A. V. Goran, JETP Lett., 90, 578 (2009).

[41] O. M. Fedorych, S. Moreau, M. Potemski, S. A. Studenikin, T. Saku, and Y. Hirayama, Intern. Journ. Mod. Phys. 23, 2698 (2009).

[42] J. H. Smet, B. Gorshunov, C. Jiang, L. Pfeiffer, K. West, V. Umansky, M. Dressel, R. Meisels, F. Kuchar, and K. von Klitzing, Phys. Rev. Lett. 95, 116804 (2005).

[43] I. V. Andreev, V. M. Muravev, I. V. Kukushkin, J. H. Smet, K. von Klitzing, and V. Umanskii, JETP Lett. 88, 616 (2008).

[44] S. A. Mikhailov and N. A. Savostianova, Phys. Rev. B 74, 045325 (2006). 